\title{
Revealing G150.3+4.5 as a dynamically young supernova remnant with gamma-ray data
}

\section{Justine Devin, ${ }^{a *}$ Marianne Lemoine-Goumard, ${ }^{a}$ Marie-Hélène Grondin, ${ }^{a}$ Daniel Castro, ${ }^{b}$ Jean Ballet, ${ }^{c}$ Jamie Cohen ${ }^{b}$ and John W. Hewitt ${ }^{d}$ on behalf of the Fermi-LAT Collaboration}

${ }^{a}$ Univ. Bordeaux, CNRS, CENBG, UMR 5797, F-33170 Gradignan, France

${ }^{b}$ Harvard-Smithsonian Center for Astrophysics,60 Garden Street, Cambridge, MA 02138, USA

${ }^{c}$ Laboratoire AIM, CEA-IRFU/CNRS/Université Paris Diderot, Département d'Astrophysique, CEA Saclay, 91191 Gif sur Yvette, France

${ }^{d}$ University of North Florida, Department of Physics, 1 UNF Drive, Jacksonville, FL 32224, USA

E-mail: jdevin.phys@gmail.com, devin@apc.in2p3.fr

Supernova remnants (SNRs) are considered one of the best candidates to accelerate the bulk of Galactic cosmic rays. Studying gamma-ray emission from young SNRs allows us to understand the nature of accelerated particles and the maximum energy they can reach. The SNR G150.3+4.5 was detected in radio and exhibits a shell-like morphology with an angular size of $3^{\circ}$, suggesting either an old or a nearby SNR. We present a spectro-morphological analysis of G150.3+4.5 with Fermi-LAT data that reveals emission up to hundreds of GeV. We estimate the distance and the surrounding density of the SNR to understand its evolutionary stage. We find that G150.3+4.5 is spectrally similar to the young shell-type SNRs observed with the Fermi-LAT such as RX J1713.7-3946 or Vela Junior. The broadband nonthermal emission of G150.3+4.5 is explained by a leptonic scenario that implies particle acceleration at least up to $\mathrm{TeV}$ energies, making G150.3+4.5 a new dynamically young SNR.

$37^{\text {th }}$ International Cosmic Ray Conference (ICRC 2021)

July 12th - 23rd, 2021

Online - Berlin, Germany

\footnotetext{
${ }^{*}$ Presenter
} 


\section{Introduction}

The supernova remnant (SNR) G150.3+4.5 was first considered an SNR candidate due to the detection of the southeastern part of the radio shell with a nonthermal spectrum [7]. Using Urumqi $6 \mathrm{~cm}$ survey data, [6] reported the discovery of a $2.5^{\circ}$ wide by $3^{\circ}$ high synchrotron emitting, shelllike object. This large extent suggests either an old or a nearby (and young) SNR. Extended $\gamma$-ray emission towards G150.3+4.5 was reported in the Fermi Galactic Extended Source catalog [3] and this source is contained in the latest Fermi-LAT catalog [4FGL, 1], named 4FGL J0427.2+5533e, whose emission is described by a logarithmic parabola (LP) spectrum.

\section{Fermi-LAT data analysis}

We used $~ 10.5 \mathrm{yr}$ of Fermi-LAT data (from August 4, 2008 to January 31, 2019) and we performed a binned likelihood analysis within a $23^{\circ} \times 23^{\circ}$ region centered on the SNR G150.3+4.5. The morphological and spectral analyses were performed from $1 \mathrm{GeV}$ to $3 \mathrm{TeV}$ and from $300 \mathrm{MeV}$ to $3 \mathrm{TeV}$, respectively. The Summedlikelihood method was used to simultaneously fit events with different angular reconstruction quality (PSF event type). We used the SOURCE event class and a pixel size of $0.1^{\circ}$ and eight (ten) energy bins per decade for the morphological (spectral) analysis. The source model contains the 4FGL sources located within a $20^{\circ}$ radius from G150.3+4.5, and we added three point sources where the Test Statistic TS $=2 \times\left(\log \mathcal{L}_{1}-\log \mathcal{L}_{0}\right)^{1}$ exceeded 25 . The spectral parameters of the sources were fit simultaneously with the Galactic and isotropic diffuse emissions ${ }^{2}$.

\subsection{Morphological analysis}

In the 4FGL catalog a point source (4FGL J0426.5+5434) is located in the southern part of the SNR with a LP spectrum. We performed the morphological analysis from $1 \mathrm{GeV}$ to $3 \mathrm{TeV}$, with the free parameters in the model being the normalization of the sources located closer than $5^{\circ}$ from G150.3+4.5, of the Galactic and isotropic diffuse emissions and the spectral parameters of G150.3+4.5 and 4FGL J0426.5+5434. We first localized the position of 4FGL J0426.5+5434 and we tested its extension by localizing a 2D symmetric Gaussian. We found that 4FGL J0426.5+5434 is not significantly extended with $\mathrm{TS}_{\mathrm{ext}}=7.7^{3}$. To describe the emission of G150.3+4.5, we tested different spatial models: a uniform disk, a 2D symmetric Gaussian and the Urumqi $6 \mathrm{~cm}$ map of the SNR. Table 1 reports the best-fit positions and extensions of the spatial models, with the associated TS and $\mathrm{TS}_{\text {ext }}$ value. The Akaike criterion [4] indicates a preference for the 2D symmetric Gaussian model compared to the uniform disk model $\left(\Delta \mathrm{TS}_{\text {Disk } \rightarrow \text { Gaussian }}=29.2\right)$. Since the residual TS map after fitting the radio template shows significant emission in the inner part of the SNR, we tested a two-component model involving the radio map of the SNR and a 2D symmetric Gaussian. The best-fit spatial parameters of the Gaussian component were found to be similar to those listed in Table 1 (in a one-component model) indicating that the radio template does not fit the $\gamma$-ray

\footnotetext{
${ }^{1} \mathcal{L}_{1}$ and $\mathcal{L}_{0}$ are the likelihood obtained with the model including and excluding the source, respectively.

${ }^{2}$ We used the file gll_iem_v07.fits and the isotropic templates derived using the preliminary inflight corrected version of the P8R3_V2 instrument response functions and extrapolated up to $3 \mathrm{TeV}$.

${ }^{3}$ Here $\mathcal{L}_{1}$ and $\mathcal{L}_{0}$ are the likelihood obtained with the extended and point-source model respectively.
} 


\begin{tabular}{lccccccc}
\hline \hline & $\operatorname{RA}_{\mathrm{J} 2000}\left(^{\circ}\right)$ & $\operatorname{Dec}_{\mathrm{J} 2000}\left(^{\circ}\right)$ & $\sigma$ or $r\left(^{\circ}\right)$ & $r_{68}\left(^{\circ}\right)$ & $\mathrm{TS}$ & $\mathrm{TS}_{\text {ext }}$ & $\mathrm{N}_{\text {dof }}$ \\
\hline Gaussian & $66.425 \pm 0.060$ & $55.371 \pm 0.056$ & $0.900_{-0.028}^{+0.029}$ & $1.359_{-0.043}^{+0.044}$ & 624.2 & 549.8 & 6 \\
Disk & $66.555 \pm 0.172$ & $55.314 \pm 0.150$ & $1.497_{-0.028}^{+0.032}$ & $1.235_{-0.023}^{+0.026}$ & 595.0 & 520.7 & 6 \\
Urumqi 6 cm map & - & - & - & - & 361.7 & 287.3 & 3 \\
\hline
\end{tabular}

Table 1: Best-fit positions and extensions $(1 \mathrm{GeV}-3 \mathrm{TeV})$ of $\mathrm{G} 150.3+4.5$ with the associated statistical errors, using a 2D symmetric Gaussian and a uniform disk model. The TS values and the number of degrees of freedom $\left(\mathrm{N}_{\mathrm{dof}}\right)$ are given for the three models tested.
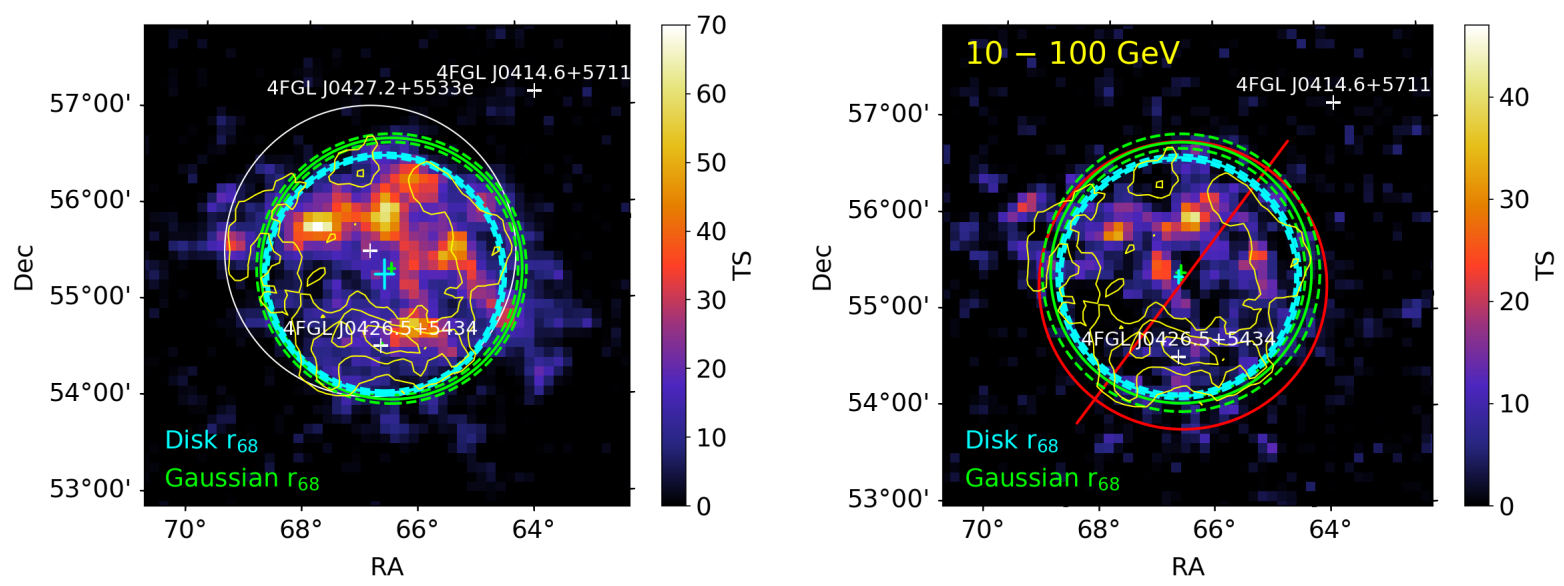

Figure 1: Residual TS maps from $1 \mathrm{GeV}$ to $3 \mathrm{TeV}$ (left) and from $10 \mathrm{GeV}$ to $100 \mathrm{GeV}$ (right) without G150.3+4.5 included in the model. The best-fit spatial models are represented. The 4FGL sources are shown in white while the red circle indicates the best-fit disk found between $1 \mathrm{GeV}$ and $3 \mathrm{TeV}$. The Urumqi $6 \mathrm{~cm}$ radio contours (at 6,11 , and $16 \mathrm{mK} \mathrm{T}_{\mathrm{B}}$ ) are overlaid in yellow [6].

morphology. Figure 1 (left) depicts the residual TS map without G150.3+4.5 included in the model with the best-fit spatial models and the radio contours overlaid. The radio emission is spatially coincident with the $\gamma$-ray emission, although the brightest parts of the radio and $\gamma$-ray emission do not arise from the same region.

We also investigated a possible energy-dependent morphology by fitting a 2D symmetric Gaussian and a uniform disk models between 1-10 GeV and 10-100 GeV. We found that the bestfit spatial parameters from $10 \mathrm{GeV}$ to $100 \mathrm{GeV}$ are very similar to those found between $1 \mathrm{GeV}$ and $3 \mathrm{TeV}$ (Figure 1, right). The spatial extents between $1-10 \mathrm{GeV}\left(r_{68}=1.26^{\circ}{ }_{-0.08^{\circ}}^{+0.00^{\circ}}\right.$ for the Gaussian model $)$ and $10-100 \mathrm{GeV}\left(r_{68}=1.36^{\circ}{ }_{-0.06^{\circ}}\right)$ are compatible within statistical errors, indicating that the $\gamma$-ray morphology does not shrink at higher energies.

\subsection{Spectral analysis}

Using the Gaussian and the disk models found in Section 2.1, we performed a spectral analysis from $300 \mathrm{MeV}$ to $3 \mathrm{TeV}$. In the 4FGL catalog, the emission from G150.3+4.5 and 4FGL J0426.5+5434 is described by a LP such as

$$
\frac{d N}{d E}=N_{0} \times\left(\frac{E}{E_{0}}\right)^{-\left(\alpha+\beta \log \frac{E}{E_{0}}\right)}
$$


We first replaced the LP spectrum of 4FGL J0426.5+5434 by a power law with an exponential cutoff that did not significantly improve the fit. Keeping the LP spectrum for 4FGL J0426.5+5434, we tested a power-law (PL) spectrum for G150.3+4.5 and we obtained a significant curvature of the spectrum with $\Delta \mathrm{TS}_{\mathrm{PL} \rightarrow \mathrm{LP}}=23.3(\sim 4.8 \sigma)$. A broken power law did not significantly improve the fit compared to the LP spectrum. The best-fit spectrum of G150.3+4.5 and 4FGL J0426.5+5434 is thus a LP. We took into account the systematic uncertainties related to the modeling of the Galactic diffuse emission, the spatial model used and the effective area. The spectrum of G150.3+4.5 is hard, with $\alpha=1.62 \pm 0.04_{\text {stat }} \pm 0.23_{\text {syst }}$ at $E_{0}=9.0 \mathrm{GeV}$, similar to the value obtained for young TeV shell-type SNRs such as RX J1713.7-3946 or RCW 86 [2]. With a soft spectral index $\alpha=$ $2.48 \pm 0.08_{\text {stat }} \pm 0.10_{\text {syst }}$ at $E_{0}=651.2 \mathrm{MeV}$ and a large curvature $\left(\beta=0.41 \pm 0.08_{\text {stat }} \pm 0.12_{\text {syst }}\right)$, 4FGL J0426.5+5434 has a spectrum reminiscent of a pulsar. No pulsations were found from this source with the 100-m Effelsberg radio telescope [5], making the nature of 4FGL J0426.5+5434 unclear.

We computed the spectral energy distributions (SEDs) of G150.3+4.5 and 4FGL J0426.5+5434 dividing the whole energy range into 8 bins and describing the emission of the components by a power law with fixed spectral index $(\Gamma=2)$. A flux upper limit was calculated if the TS of the source is lower than 1. Figure 2 (left) shows the SEDs of G150.3+4.5 and 4FGL J0426.5+5434, using the $2 \mathrm{D}$ symmetric Gaussian in the model. The emission at low energy is dominated by the contribution from 4FGL J0426.5+5434, while the emission from the SNR arises at higher energy. Since the spatial model used for G150.3+4.5 (a uniform disk or a 2D symmetric Gaussian) has a negligible impact on the spectral analysis (Figure 2, right), we used the morphological and spectral properties of the disk to calculate the physical parameters of the SNR and discuss the nature of the emission.
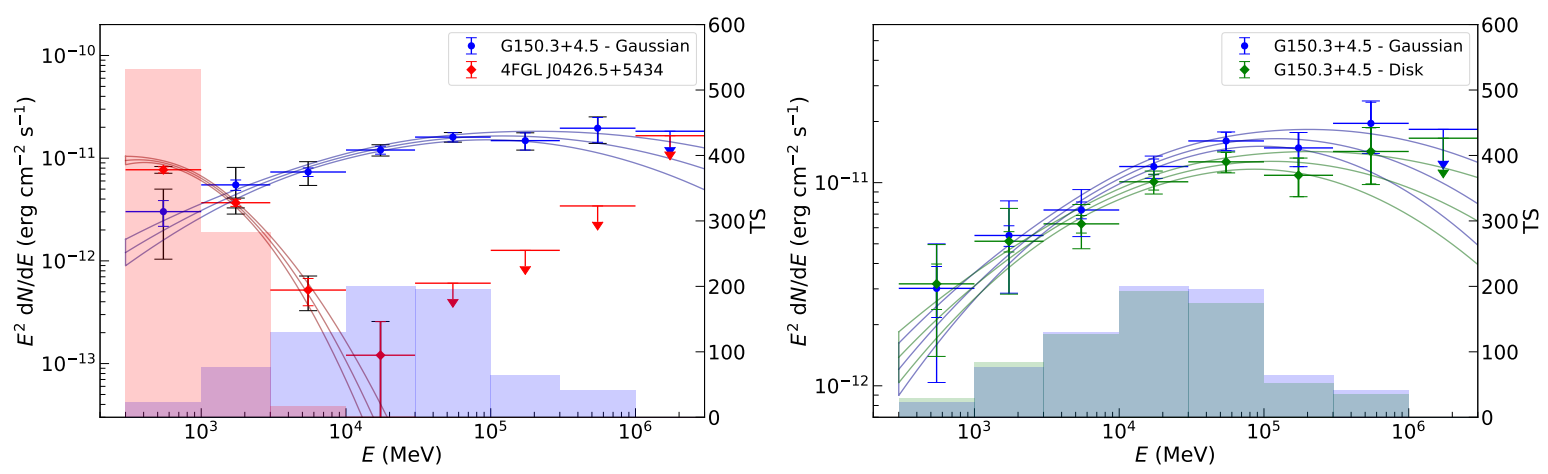

Figure 2: (Left) SEDs of G150.3+4.5 and 4FGL J0426.5+5434 with the associated statistical errors (colored bars) and the quadratic sum of the statistical and systematic errors (black bars). The shaded areas correspond to the TS value in each energy band, and the upper limits are calculated at the $95 \%$ confidence level. (Right) Comparisons of the SEDs obtained using a Gaussian and a disk model for G150.3+4.5. The colored bars represent the quadratic sum of the statistical and systematic errors.

\section{X-ray data and distance estimate}

We used archival observations from the ROSAT all-sky survey [10] to determine whether there is significant X-ray emission $(0.1-2.4 \mathrm{keV})$ in the direction of G150.3+4.5. The ROSAT data was 
extracted within a $1.3^{\circ}$ circle centered on $\mathrm{RA}=4: 27: 08.528$, $\mathrm{Dec}=+55: 27: 29.53$ with a total exposure of $19 \mathrm{ks}$. No significant thermal or nonthermal emission is detected from G150.3+4.5. To estimate the minimum distance to G150.3+4.5, we used the Sedov-Taylor self-similar solution

$$
R_{S} \approx 0.314 \times\left(\frac{E_{51}}{n_{0}}\right)^{1 / 5} t_{\mathrm{yr}}^{2 / 5} \mathrm{pc}
$$

where $E_{51}$ is the kinetic energy released by the supernova, $n_{0}$ the ambient density, and $t_{\mathrm{yr}}$ the age of the SNR in units of $10^{51} \mathrm{erg}, \mathrm{cm}^{-3}$ and years respectively. Assuming $E_{51}=1$ and knowing the angular size of G150.3+4.5, we used different combinations of distance and age to calculate the corresponding ambient density. We then modeled the emission as an absorbed thermal nonequilibrium ionization plasma for each combination of age, ambient and column densities, and electron and proton temperature. For each distance we thus calculated the corresponding column density, and we obtained an upper limit on the ambient density. The maximum ambient density allowed by ROSAT data is $n_{0}=3.6 \times 10^{-3} \mathrm{~cm}^{-3}$. Since an SNR at Dec $\sim 55^{\circ}$ and younger than $1 \mathrm{kyr}$ would have been reported in historical records, we imposed a lower limit on the age of the SNR of $t=1 \mathrm{kyr}$, giving a minimum distance of $d=0.7 \mathrm{kpc}$ for an ambient density consistent with ROSAT data $\left(n_{0}=1.5 \times 10^{-3} \mathrm{~cm}^{-3}\right.$ at that distance). To constrain the maximum distance, we imposed a Mach number $\mathcal{M}^{2}>10$ (equivalent to a particle spectral index $p<2.4$ ). With the sound speed $c_{s}^{2}=$ $\frac{\gamma P_{\text {ISM }}}{\rho_{0}}\left(\gamma=5 / 3\right.$ being the adiabatic index and $P_{\text {ISM }}$ the interstellar medium pressure $)$, this condition can be written as $\rho_{0} v_{s}^{2}>10 \rho_{0} c_{s}^{2}=10 \gamma P_{\text {ISM }}$. In the Sedov model, this condition translates into a relation among the physical radius of the SNR, the interstellar medium pressure, and the kinetic energy released by the supernova

$$
R_{S}<1.69 \times\left(\frac{P_{\mathrm{ISM}}}{k} \times \frac{1}{E}\right)^{-1 / 3} \mathrm{kpc} .
$$

Assuming $E_{51}=1$ and taking $\left(P_{\mathrm{ISM}} / k\right)=2.3 n_{0} T_{\mathrm{ISM}}=3000 \mathrm{~K} \mathrm{~cm}^{-3}$, we found $R_{s}<117.2 \mathrm{pc}$, giving $d<4.5 \mathrm{kpc}$ for $r=1.497^{\circ}$.

\section{Discussion}

The spatial correlation between the radio and the $\gamma$-ray emissions, together with the lack of a $\gamma$-ray morphology shrinking at higher energies, points toward an SNR scenario. This is supported by the radio shell-like appearance and the nonthermal radio spectrum similar to that obtained in SNRs.

Figure 3 (left) depicts the SEDs of several Fermi-LAT detected SNRs and shows that G150.3+4.5 is spectrally similar to the dynamically young and shell-type SNRs. For the minimum distance, the luminosity of G150.3+4.5 between $100 \mathrm{MeV}$ and $100 \mathrm{GeV}$ is low and similar to that obtained for young SNRs, while for the maximum distance the luminosity is closer to that of SNRs interacting with molecular clouds (Figure 3, right). However, there is no hint of an interaction between G150.3+4.5 and a molecular cloud in the archival multiwavelength data. The hard spectral shape of G150.3+4.5 and its likely low luminosity supports the dynamically young and non-interacting SNR scenario, and therefore a near distance.

To understand the origin of the $\gamma$-ray emission, we performed multiwavelength modeling using the Naima package [11] in a one-zone model assumption. We used the radio fluxes derived in [7] 

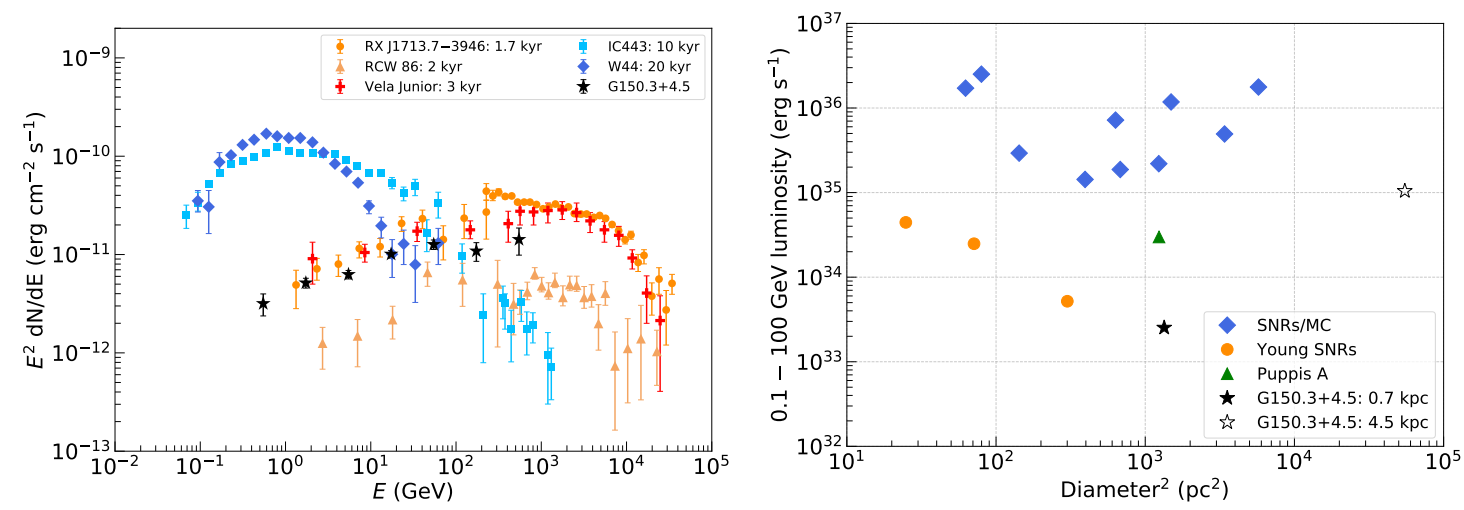

Figure 3: (Left) SEDs of several Fermi-LAT SNRs interacting with molecular clouds (blue tone colors) and being dynamically young (orange tone colors), with that of G150.3+4.5. (Right) Luminosity of several Fermi-LAT observed SNRs plotted against their diameter squared. Black stars correspond to the luminosity of G150.3+4.5 using a distance of $0.7 \mathrm{kpc}$ and $4.5 \mathrm{kpc}$. The figure is reproduced from [9] with the addition of $\mathrm{G} 150.3+4.5$.

and the $\gamma$-ray spectrum obtained with the disk model, and we factored the X-ray absorption into the model, allowing the use of the robust upper limit on the absorbed X-ray flux obtained with ROSAT data. Since the radio fluxes were derived in a region of $\sim 2.14^{\circ} \times 0.63^{\circ}$ [7] that does not encompass the entire radio SNR $\left(\sim 3^{\circ} \times 2^{\circ}\right)$, we used these data points as lower limits. We described the proton spectrum by a power law with an exponential cutoff and the electron spectrum by a broken power law with an exponential cutoff (the break being due to synchrotron cooling). Since the radio spectral index is $\alpha=-0.38 \pm 0.10$, we fixed the electron spectral index to $s_{\mathrm{e}, 1}=1.8$, giving $s_{\mathrm{e}, 2}=2.8$, and we used the same value for the proton spectral index $s_{\mathrm{p}}=1.8$. For the inverse Compton scattering on photon fields, we considered the cosmic microwave background, the infrared and optical emissions for which the temperature and energy density are estimated with the GALPROP ${ }^{1}$ code [8]. For the minimal distance of $d=0.7 \mathrm{kpc}$, the temperature and energy density of the infrared and optical emissions are $T_{\mathrm{IR}}=25.2 \mathrm{~K}, \mathrm{U}_{\mathrm{IR}}=0.34 \mathrm{eV} \mathrm{cm}^{-3}$ and $T_{\mathrm{opt}}=2004.9 \mathrm{~K}, \mathrm{U}_{\mathrm{opt}}=0.52 \mathrm{eV} \mathrm{cm}^{-3}$. We used the ambient density of $n_{0}=1.5 \times 10^{-3} \mathrm{~cm}^{-3}$ derived in Section 3, and the column density of $N_{H}=5.23 \times 10^{20} \mathrm{~cm}^{-2}$ (corresponding to a distance of $d=0.7 \mathrm{kpc}$ ) to model the absorbed X-ray nonthermal emission. With a total proton energy of $W_{\mathrm{p}}=10^{50} \mathrm{erg}$, the data can be reproduced by a leptonic scenario with a downstream magnetic field of $B=5 \mu \mathrm{G}$, an electron-to-proton ratio of $K_{\text {ep }}$ $=1 \times 10^{-3}$ and an acceleration of particles up to $E_{\max , \mathrm{e}}=E_{\max , \mathrm{p}}=5.2 \mathrm{TeV}$ (Figure 4). Given the low magnetic field and age of the SNR $(t=1 \mathrm{kyr})$, electrons do not suffer significantly from synchrotron cooling so there is no break in the electron spectrum. For the maximum distance of $d=4.5 \mathrm{kpc}$, the data can be reproduced with a similar model $\left(B=5 \mu \mathrm{G}\right.$ and $\left.E_{\text {max,e }}=E_{\text {max,p }} \sim 5 \mathrm{TeV}\right)$ taking $K_{\text {ep }}$ $=5 \times 10^{-2}$. We did not consider the hadron-dominated scenario due to the low maximum ambient density allowed by ROSAT data (Section 3), which would lead to an unrealistic value of $W_{\mathrm{p}}$, and due to the lack of any hint of interaction of the SNR with interstellar material.

1https://galprop.stanford.edu 


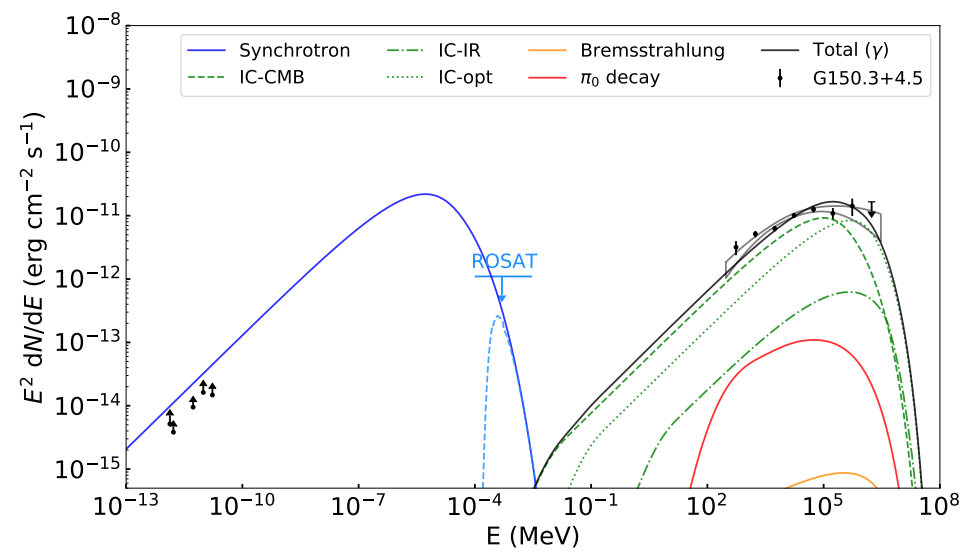

Figure 4: Broadband nonthermal modeling of the SNR G150.3+4.5 in a leptonic scenario using a distance of $d=0.7 \mathrm{kpc}$. The radio fluxes, which are near lower limits, are extracted from [7]. The ROSAT upper limit on the absorbed flux has to be compared to the absorbed X-ray synchrotron model (dashed blue curve).

\section{Conclusion}

We performed a spectro-morphological analysis of G150.3+4.5 using Fermi-LAT data. The $\gamma$-ray emission is extended from $1 \mathrm{GeV}$ to $3 \mathrm{TeV}$ and spatially coincident with the radio emission of the SNR. The spectral analysis from $300 \mathrm{MeV}$ to $3 \mathrm{TeV}$ reveals a hard spectrum for G150.3+4.5, similar to that obtained for young shell-type SNRs, and a spectrum reminiscent of a pulsar for 4FGL J0426.5+5434 which is located in the southern part of the SNR. We found no significant thermal and nonthermal X-ray emission towards G150.3+4.5. We estimated minimal and maximal distances of $0.7 \mathrm{kpc}$ and $4.5 \mathrm{kpc}$ and we compared the luminosity of G150.3+4.5 with that of other Fermi-LAT observed SNRs. We found that a scenario involving a dynamically young and non-interacting SNR located at a near distance is favored. We modeled the broadband nonthermal spectrum of G150.3+4.5 with a leptonic scenario, implying a downstream magnetic field of $B=5$ $\mu \mathrm{G}$ and acceleration of particles at least up to TeV energies. Deeper multiwavelength observations would be required to better constrain the broadband nonthermal emission from G150.3+4.5. In particular, observations at very-high energy would allow to derive the maximum energy reached by particles in this SNR.

\section{Acknowledgements}

We thank X. Y. Gao and J. L. Han for providing us the Urumqi $6 \mathrm{~cm}$ map of the SNR. JD, MLG and MHG acknowledge support from Agence Nationale de la Recherche (grant ANR- 17-CE310014). The Fermi LAT Collaboration acknowledges generous ongoing support from a number of agencies and institutes that have supported both the development and the operation of the LAT as well as scientific data analysis. These include the National Aeronautics and Space Administration and the Department of Energy in the United States, the Commissariat à l'Energie Atomique and the Centre National de la Recherche Scientifique / Institut National de Physique Nucléaire et de Physique des Particules in France, the Agenzia Spaziale Italiana and the Istituto Nazionale di Fisica Nucleare in Italy, the Ministry of Education, Culture, Sports, Science and Technology (MEXT), 
High Energy Accelerator Research Organization (KEK) and Japan Aerospace Exploration Agency (JAXA) in Japan, and the K. A. Wallenberg Foundation, the Swedish Research Council and the Swedish National Space Board in Sweden. Additional support for science analysis during the operations phase is gratefully acknowledged from the Istituto Nazionale di Astrofisica in Italy and the Centre National d'Études Spatiales in France. This work performed in part under DOE Contract DE-AC02-76SF00515.

\section{References}

[1] Abdollahi, S., Acero, F., Ackermann, M., et al. 2020, , 247, 33

[2] Acero, F., Lemoine-Goumard, M., Renaud, M., et al. 2015, , 580, A74

[3] Ackermann, M., Ajello, M., Baldini, L., et al. 2017, , 843, 139

[4] Akaike, H. 1974, IEEE Transactions on Automatic Control, 19, 716

[5] Barr, E. D., Guillemot, L., Champion, D. J., et al. 2013, , 429, 1633

[6] Gao, X. Y. \& Han, J. L. 2014, , 567, A59

[7] Gerbrandt, S., Foster, T. J., Kothes, R., Geisbüsch, J., \& Tung, A. 2014, , 566, A76

[8] Porter, T. A., Moskalenko, I. V., Strong, A. W., Orlando, E., \& Bouchet, L. 2008, , 682, 400

[9] Thompson, D. J., Baldini, L., \& Uchiyama, Y. 2012, Astroparticle Physics, 39, 22

[10] Voges, W., Aschenbach, B., Boller, T., et al. 1999, VizieR Online Data Catalog, 9010

[11] Zabalza, V. 2015, in 34th International Cosmic Ray Conference (ICRC2015), Vol. 34, 922 